\title{
Stem Cell Transplantation in Multiple Sclerosis: Sharing the Experience
}

\author{
Tatyana Ionova
}

(C) Humana Press 2009

The International Symposium "Stem Cell Transplantation in Multiple Sclerosis: Sharing the Experience" will be held on October 5th, 2009 in Moscow, Russia. Symposium organizers are Pirogov National Medical Surgical Center, National Center for Research and Treatment of Autoimmune Diseases, Russian Cooperative Group for Cellular Therapy, and New Jersey Center for Quality of Life and Health Outcomes Research.

The symposium will be the first special meeting fully meant to discuss the state-of-the-art and perspectives of the new and quite promising method of multiple sclerosis treatment-high-dose immunosuppressive therapy+autologous hematopoietic stem cell transplantation. It intends to share the newly acquired knowledge in the field to discuss challenges and perspectives of the method, and to develop collaborative projects. The topics to be covered within the symposium include:

- Regimens of conditioning: Immunoablation or immunosupression?

\footnotetext{
T. Ionova $(\bowtie)$

Pirogov National Medical Surgical Center,

70 Nighnyaya Pervomaiskaya str.,

105107 Moscow, Russia

e-mail: tation16@gmail.com
}

- Types of transplantation: autologous or allogenic?

- Posttransplant immunological reconstitution. Side effects

- Outcome measures: clinical, imaging, patient-reported outcomes

- Posttransplant neurorehabilitation

- Long-term follow-up results

- Proposals for cooperative studies

Neurologists, immunologists, transplantologists, hematologists, and specialists in stem cell research are invited to participate in the symposium.

Key dates:

1 May 2009-Deadline for abstract submission 1 April 2009-Deadline for early registration

Symposium Organizers Contact Information:

Tel: +7 4954634923 or +7 9627101711

E-mails: nqolc@yandex.ru and qlife@rambler.ru

Web-site: http://www.stemcellms.ru 